\title{
Calibration of Acoustic Emission System for Materials Characterization
}

\author{
S. V. Ranganayakulu ${ }^{1}$, B.Samrat Goud ${ }^{1}$, P V Sastry ${ }^{2, *}$, B. Ramesh Kumar ${ }^{3}$ \\ ${ }^{1}$ Centre for Non Destructive Evaluation, Guru Nanak Institutions Technical Campus, India \\ ${ }^{2}$ R\&D Division, Bharat Heavy Electricals Limited, India \\ ${ }^{3}$ Prototype VV \&Pellet Injector Division, Institute for Plasma Research, India
}

Copyright $(\mathcal{C} 2015$ by authors, all rights reserved. Authors agree that this article remains permanently open access under the terms of the Creative Commons Attribution License 4.0 International License

\begin{abstract}
Acoustic Emission (AE) is one of the versatile tools to study the materials and associated defects non-destructively under dynamic or static stress load conditions. The experiments planned to study Acoustic Emission system have to be calibrated with various resonant frequency band sensors and stress levels appropriately. This practice covers requirements for the calibration of acoustic emission sensors. The calibration yields the frequency response of a sensor to acoustic waves of the type which normally comes upon in acoustic emission work. The source producing the signal is used for the calibration that increases on the same surface of the test block as the sensor under testing. The calibration represents primarily of the sensor sensitivity to Rayleigh waves. The sensitivity of the sensor is determined for excitation within the range of $1000 \mathrm{KHz}$ to $1 \mathrm{KHz}$. The sensitivity value is usually determined at frequency approximately $1 \mathrm{KHz}$ apart. The AE sources are generally from high intensity stress zones, cracks or defects in the materials under mechanical loads. The acoustic sound signals generated in the dynamic load conditions like burst or continuous emissions must be correlated with parameters such as velocity, time and displacement within the testing system. AE - 4 Channel Acoustic Emission Detection System (procured from Physical Acoustic Corporation, USA) with various frequency band sensors covering wide range is used in this study for the calibration procedure establishment. $\mathrm{AE}$ sensors are arranged in vector and matrix mode for signal analysis through AE parameters. In order to deduce physical description of $\mathrm{AE}$ sources from recorded waveforms, a well characterized and calibrated $\mathrm{AE}$ sensor is required for study of signal analysis. The present paper reports the estimation of the physical quantity measured by the sensor i.e., velocity of material under test. Subsequently, AE source is used for sound signal to travel in the material such that velocity of the material is calculated by manual displacement and time mode. Calibration of sensors is evaluated by shear velocity and experimental standardization studied by the arrangement of different kinds of sensors namely - R6ó, WS ó, PK15I and PK6I. The calibration procedure and standardization for testing of the materials is established and reported.
\end{abstract}

Keywords AE Parameters, Sensors, Calibration, Standardization

\section{Introduction}

Acoustic Emission technique is emerging as a powerful tool for Non - destructive evaluation $[1,2]$. AE is defined as the phenomenon; where elastic waves are generated by rapid release of energy from localized sources like places of transient relaxation of stress and strain fields. We can analyze AE signals and detect the location of discontinuities [3]. When experiment is being conducted we can face some difficulties which are caused by couplant that are used, by repeatability of emitted sound waves, force and load effect etc. These problems can be reduced but the major problem is sensitivity of sensors. Without good sensitivity of sensors we cannot get correct signals. Obviously, this may affect our conclusion of studies. Hence we have to calibrate the sensors which we are using for experimental studies prior to the conduct of experimental studies. We can start the testing after calibration and standardizing. ASTM E1106 outlines a method for primary calibration of AE sensor [4]. Here we followed Hue Nelson Shoe Method (pencil led breaking) for calibration and standardization of AE sensors. AE inspection is extremely sensitive compared to the other more familiar NDT methods $[5,6]$. If ideal conditions are met for each method the minimum detectable crack size for UT, RT and ECT methods is about $0.50 \mathrm{~mm}$ whereas AE can detect crack growth of the order of 25 microns. This corresponds to micro crack growth of the order of less than 10 microns.

\subsection{AE Sensors}

An Acoustic Emission Sensor converts the mechanical energy carried by the elastic wave into an electrical signal; the sensor is termed as transducer. The transducer most often used in AE applications is the piezoelectric transducer. This choice has been dictated by the ease with which it may be built, its inherently high sensitivity and ruggedness allows its 
use in industrial applications.

The active element of a piezoelectric transducer is a thin disk of piezoelectric material (a material which can convert mechanical deformation into electrical voltage) [7]. This disk is metalized on both faces for electrical contact mounted in a metal cylinder to provide electromagnetic interference shielding. The piezoelectric ceramics commonly used in $\mathrm{AE}$ transducers are made of small crystals of titivates and zirconates which are mixed with other materials, molded to the desired shape, and fired in a kiln. While the ceramic material is in a strong electric field and when made piezoelectric by poling, is set to be the process of heating the material above its Curie temperature.

To take advantage of the extreme sensitivity of the piezoelectric transducer, it must be attached to the material under observation in such a manner that the acoustic energy passes into the transducer with minimum loss at the transducer-material interface. The required intimate mechanical contact is achieved on flat surfaces by mechanical clamping using thin films of grease, oil or epoxy adhesive between the transducer and the material. In general, the problem of coupling is much more severe for shear wave observation because the coupling medium must be sufficiently viscous to support the shear motion.

When a sensor has simply been placed on the surface of the material containing the acoustic wave it is found that the sensor produces a very weak signal. If a thin layer of a fluid is placed between the sensor and the surface, a much larger signal is obtained. The use of some type of couplant is almost essential for the detection of low level acoustic signals. 4Channel AE setup was used to conduct the experiment along with (Fig: 1) transducers such as R6ó, WS ó, PK15I and PK6I. (Fig: 2)

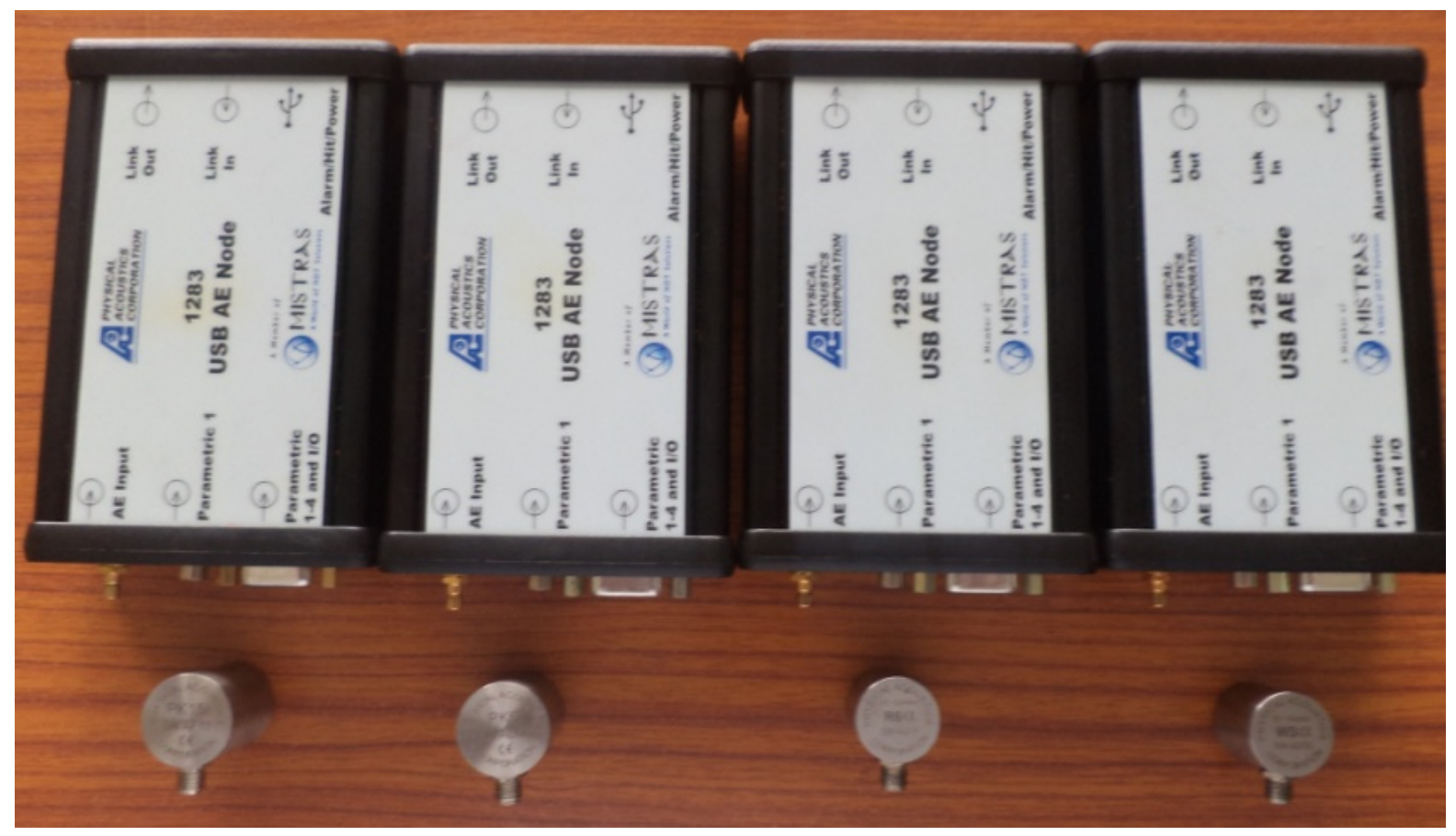

Figure 1. AE transducers with respective nodes

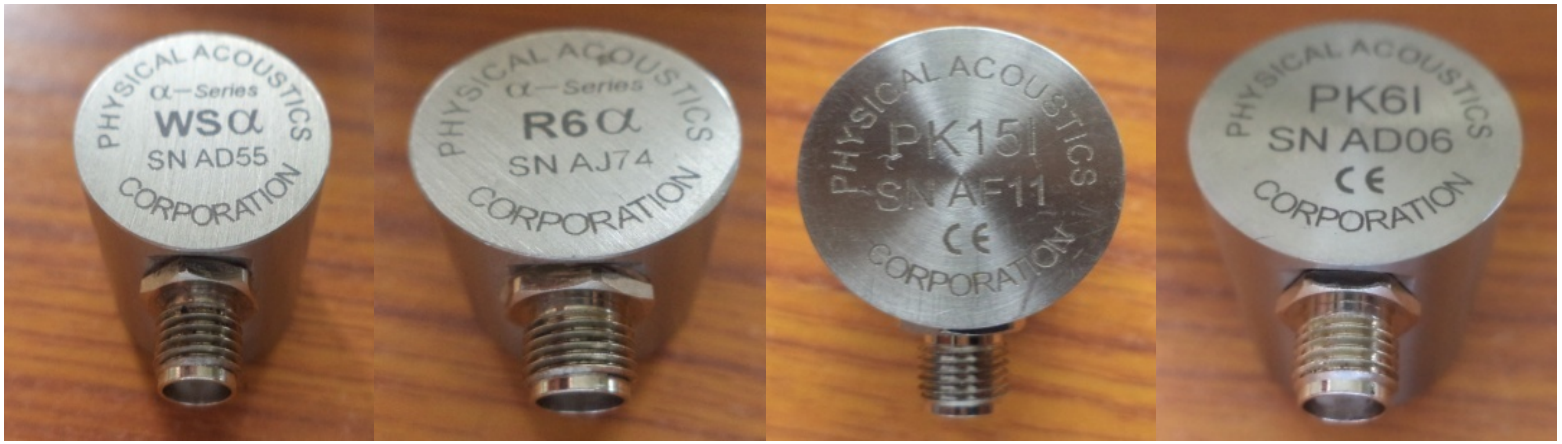

Figure 2. AE Transducers 


\subsection{Calibration Procedure}

The most widely used [8] simulated AE source is PLB (Pencil Lead Breaking). The applied force produces a local deformation that is suddenly released when lead breaks. The Hsu Pencil (named after the developer of technique) and the accessory Nielsen Shoe [9] are convenient, inexpensive aids that have been enormously valuable in practical $\mathrm{AE}$ testing. The breaking of the lead undergoes very short duration, which gives raise to a localized impulse that is quite similar to a natural Acoustic Emission source such as a crack. Furthermore the amplitude of lead break source is well within the range of typical crack source. The Hsu pencil becomes a simulated $\mathrm{AE}$ source that is used in some procedures for wide monitoring and the maximum permissible sensor spacing is based on the ability to detect lead breaking from anywhere in the inspection area. ASTM-
E 1106 standard is followed for primary calibration of Acoustic Emission Sensors.

\section{Methods of AE Sensors Calibration}

The calibration of a sensor is the measurement of its voltage output into an established electrical load for a given mechanical input. (Fig: 3 )

\subsection{Surface Calibration or Rayleigh Calibration}

The sensor and the source are located on the same plane surface of the test block (Fig: 4)

The energy at the sensor travels at the Rayleigh speed and the calibration is influenced by the aperture effect.

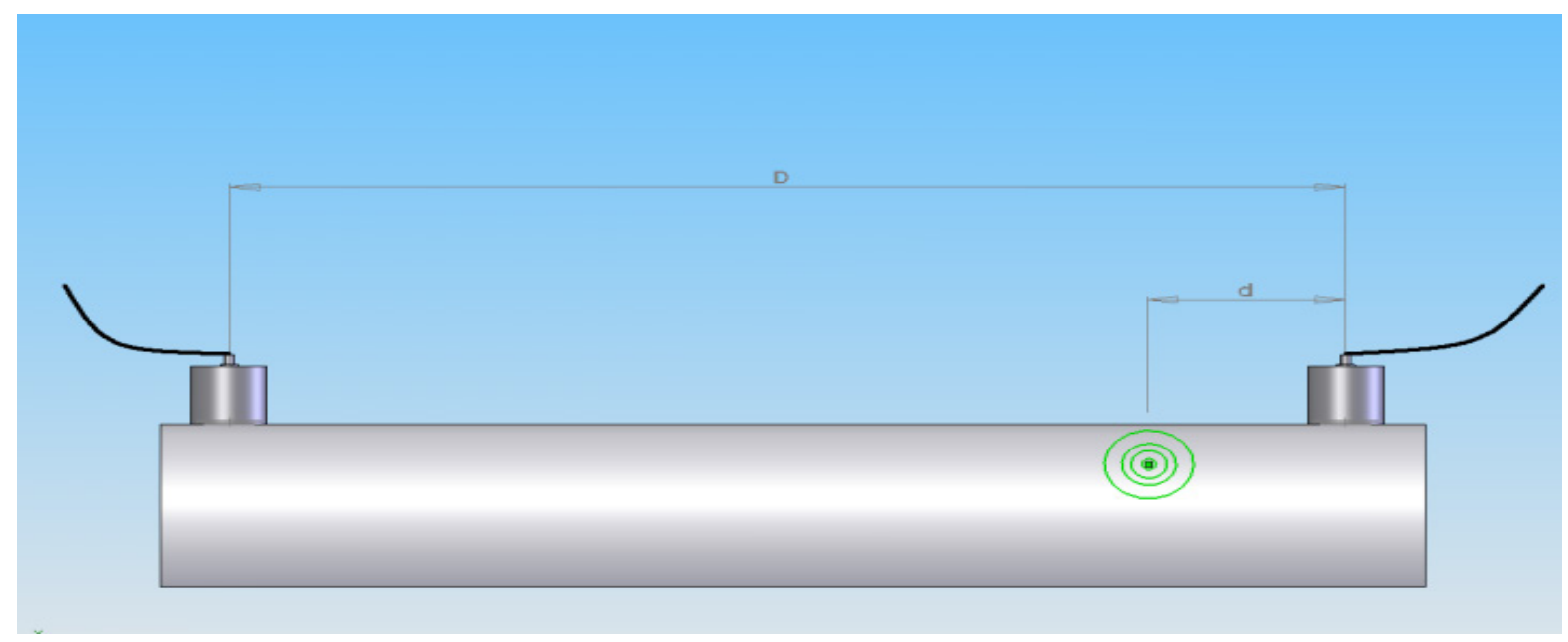

Figure 3. Arrangement of Sensors on the specimen
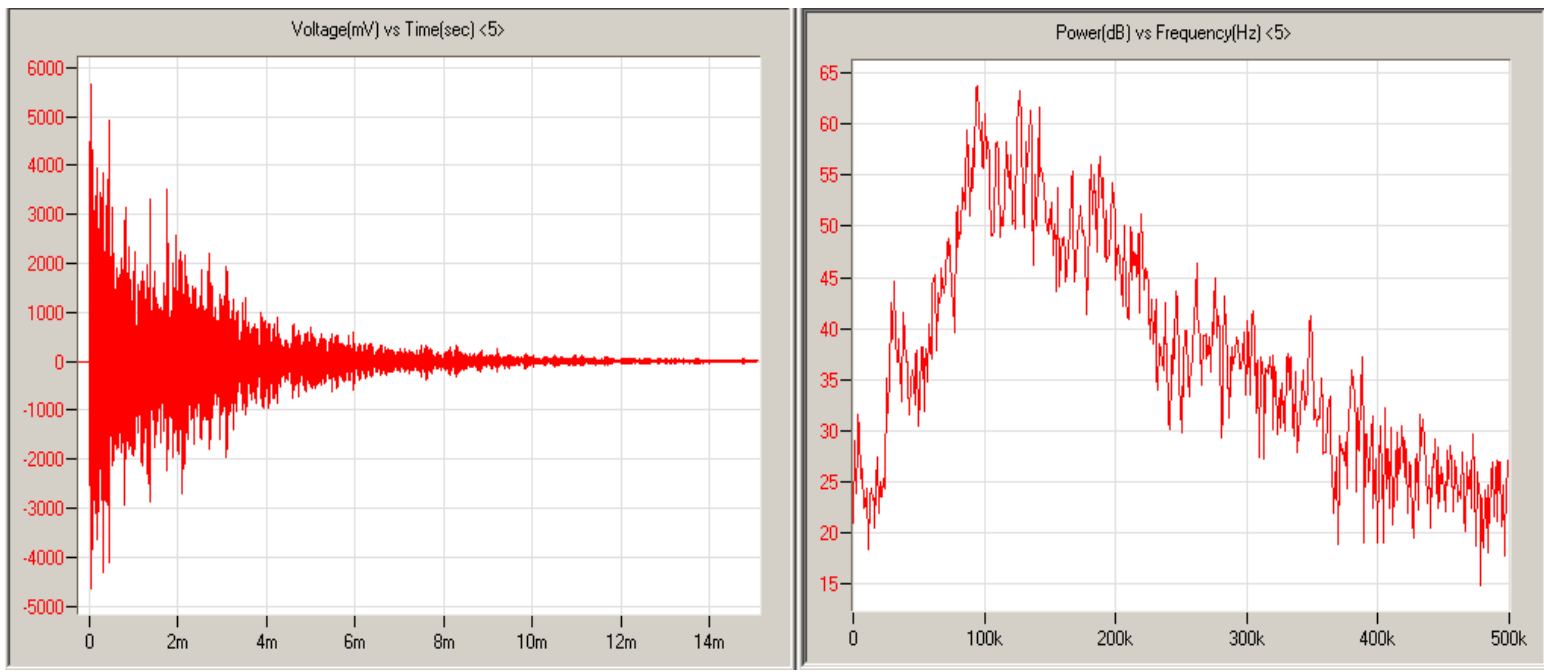

Figure 4. AE signals of Voltage vs Time and Power vs Frequency 


\subsection{Through Pulse Calibration}

The sensors and the sources are co-axially situated opposite to parallel surfaces and wave motion is free of any aperture effect. (Fig: 5)

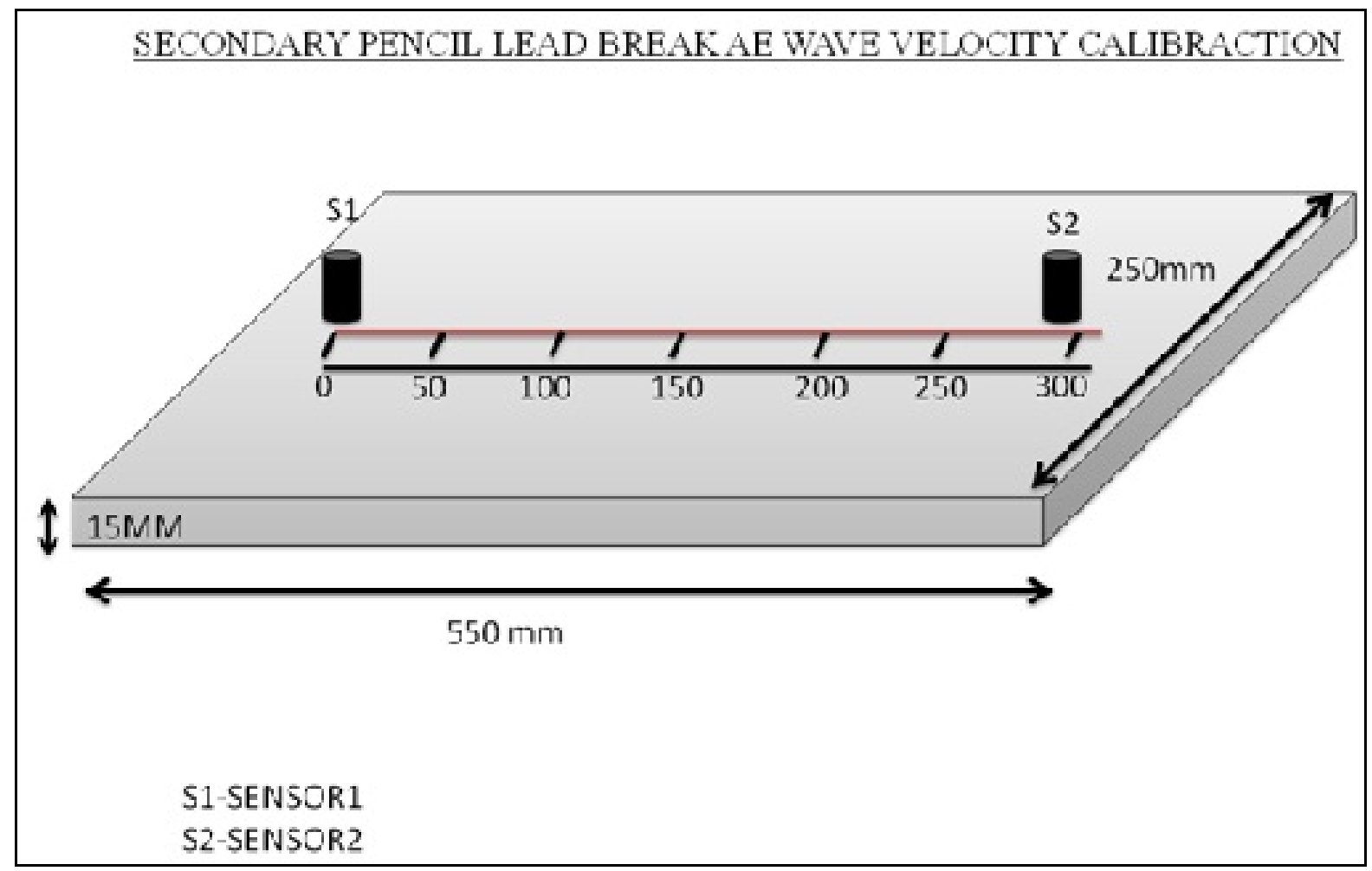

Figure 5. AE Calibration Block

\section{Pencil Lead Break Method Sample}

This test method covers the requirements for the absolute calibration of Acoustic Emission (AE) sensors. The calibration yields the frequency response of a transducer to waves on the surface of the type normally encountered in acoustic emission work. The transducer voltage response is determined to be well established dynamic displacement normal to the mounting surface. (Fig: 6)

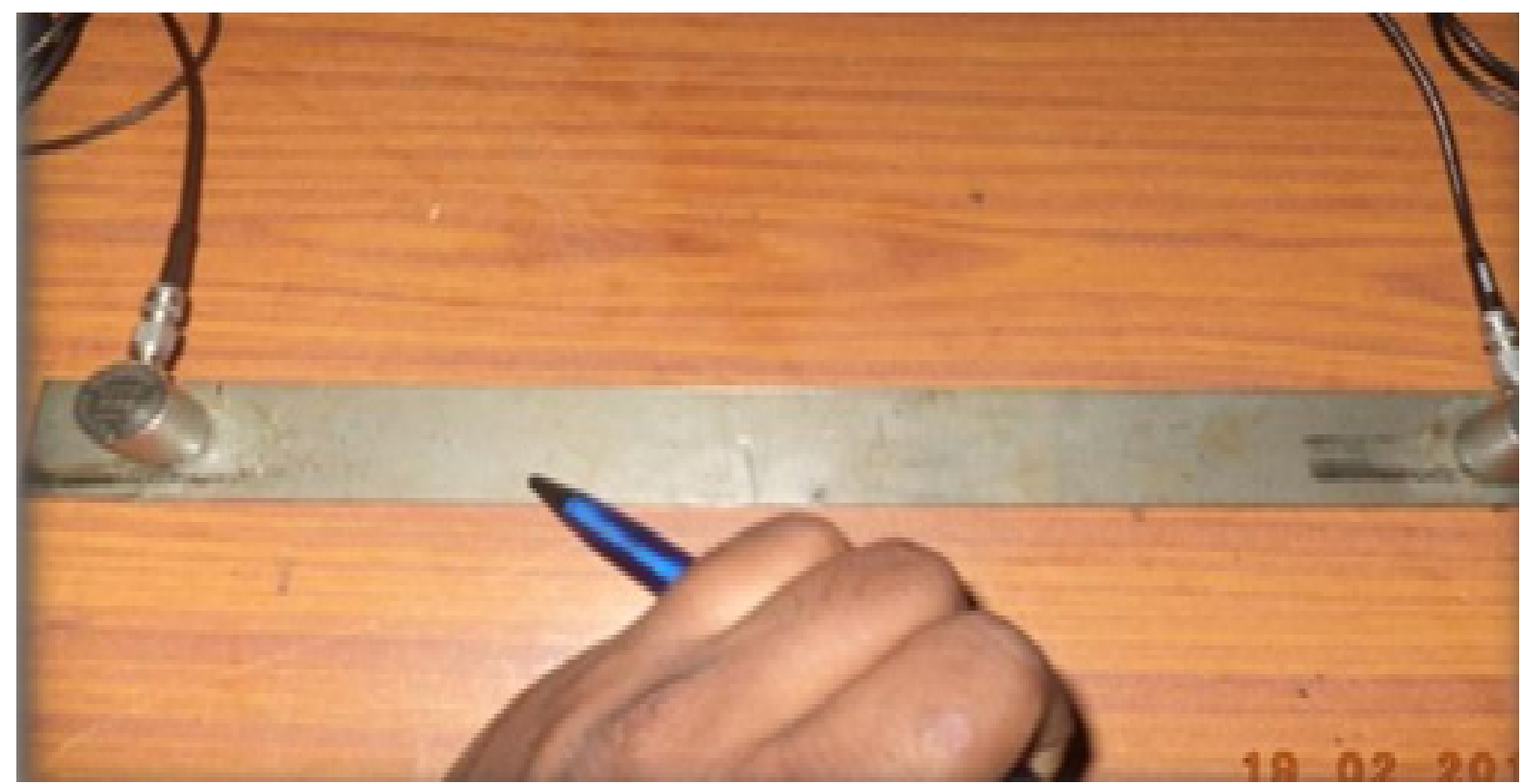

Figure 6. Pencil Lead Break Method on test sample 


\section{Sample Preparation}

The sample which is used for testing is Stainless Steel 316 to avoid distortion and interruption when conducting experiment. Initially sample was welded and then total groove is removed by machining so that sample can stick to table. The measurement of sample consists of $300 \mathrm{~mm}$ X $40 \mathrm{~mm}$ X $8 \mathrm{~mm}$. The Welding technique used is TIG welding.

Parameters maintained during the welding of stainless steel:

- Root gap: $2 \mathrm{~mm}$.

- Argon gas: 5LPH - 6 LPH.

- V- groove- $60^{0}$, No of passes: $4-5$.

- Maintained angle of filler metal is $30^{\circ}$ and torch angle is $60^{\circ}$

- 5 mins of time is taken for each sample

- Filler metal which has same composition with respect to parent metal i.e., SS material.

- Welding speed-70mm/min.

- Voltage - 400volts, Currents- 110-120 amp

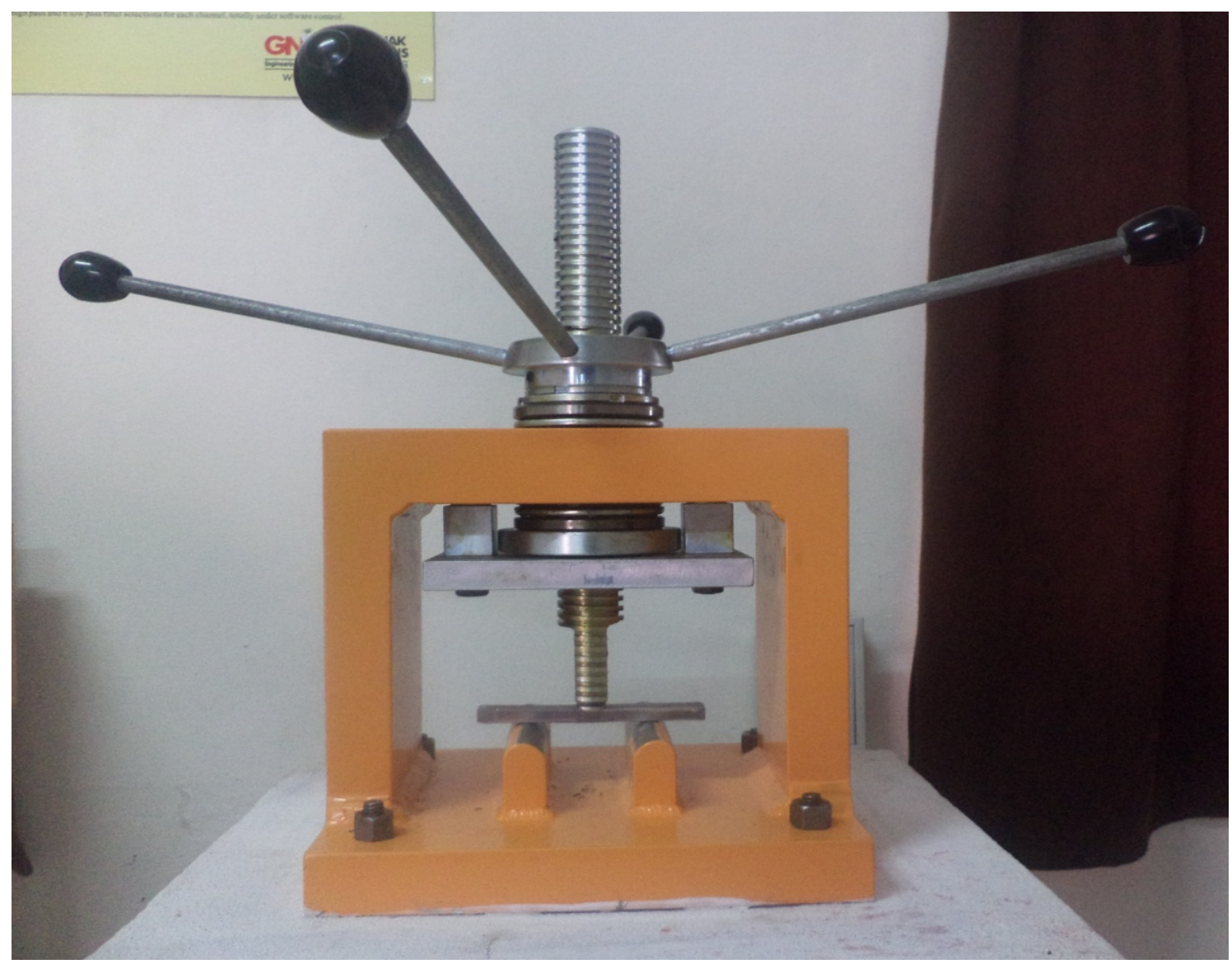

Figure 7. Mechanical Jig for apply of point load on the weld zone of the specimen

\section{Sensitivity of Specimen}

- Welded specimen dimensions are $140 * 16 * 10 \mathrm{~mm}$ which is placed on the anvil of mechanical jig for calibration.

- A point load is applied on the weld zone of the specimen (Fig: 7) and correspondingly events are observed and reported (Fig: 8)

- $\mathrm{He}-$ Nielsen method is applied for calibration of specimen.

- The digital occurrence of events are tuned with stability of the specimen at different distances 

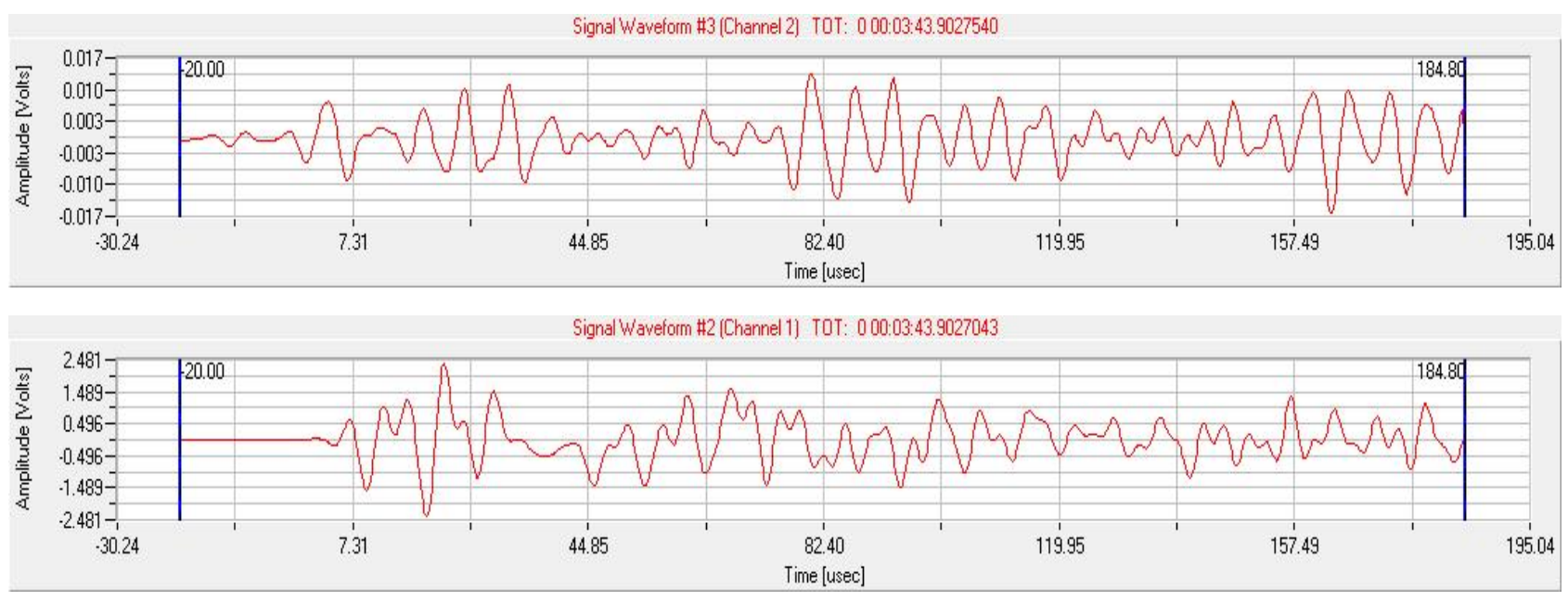

Figure 8. Amplitude vs Time from channel 2 and channel 1

\subsection{Static Condition}

Without applying any external loads (dynamic loading) the sample acoustic signals are produced with no deformation that is on sample. This type of static test is almost equal to calibration method. Similarly, formation of events is very less in static condition. Both calibration and static condition are Pencil Lead Breaking methods. Stress applied on specimen is less in static condition. So the defects or flaws present in welded region are difficult to produce the acoustic sources and the stress forms at micro levels (Fig: 9). The main advantage in static condition is to find out the source location easily when two sensors are used because the contact area between surface of specimen and lead is very less. Therefore the time arrival of each sensor is calculated and the stress affected region is found.

The main limitation in Pencil Lead Break Method is to make contact on specimen each time with same stress and same contact angle with the pencil. The Parameters play a main role in acoustic emission testing like HITS, EVENTS, AMPLITUDE, ENERGY and RISETIME. All the parameters are very low when compared to dynamic condition. As higher amplitude is the source emitted by defects.

\subsection{Dynamic Condition}

The test is carried by applying the load on the material to differentiate the results from the static condition (result). Here in dynamic condition the defects in material exhibits source (sounds) when the stress (load) is applied on material. When the deformation starts it exhibits in three different stages. In these stages the source shows high amplitude and energy values when compared to static. The load is applied in the form of tensile or compresses type. In these methods the material gets deformation due to external loads applied on it. Load can be applied on material by adopting Universal Testing Machine (UTM) through mechanical jig (prepared by use which is suitable for Acoustic Emission Testing) (Fig. ). This special jig was designed for ease of loading and to keep the unwanted external noise to the minimum. This jig has provision for placement of the samples on an anvil at the lower side and in the center. (Fig: 10)

This anvil has two vertical supports with rounded tops on which the sample can be placed and pressed to obtain deformations. A mandrill attached to a screw loading system can be lowered on the material by using jig at the centre of the welded region. Minimum contact area is possible by this jig method to reduce the formation of unwanted events. 

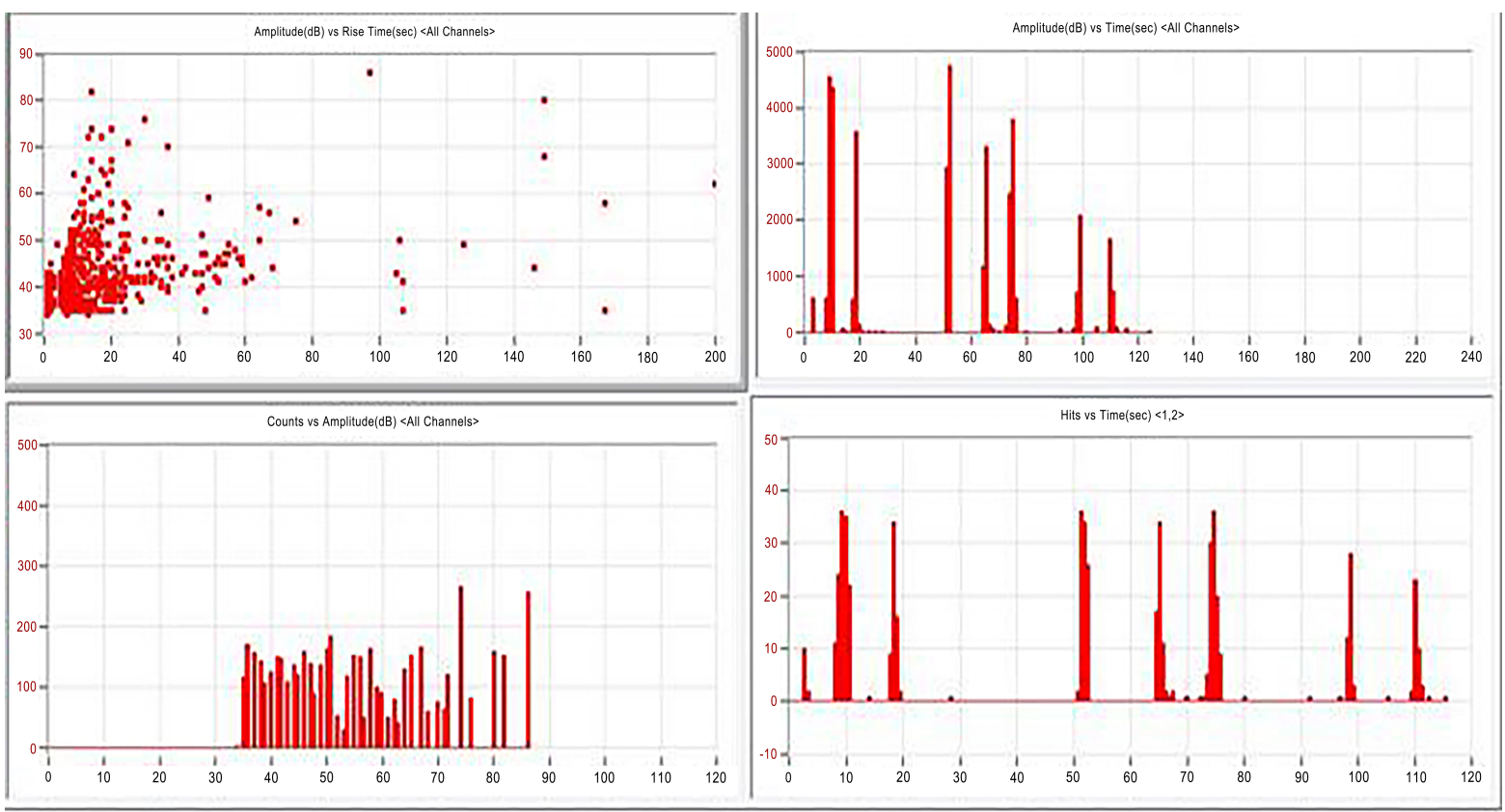

Figure 9. AE signals of the sample during Static Condition
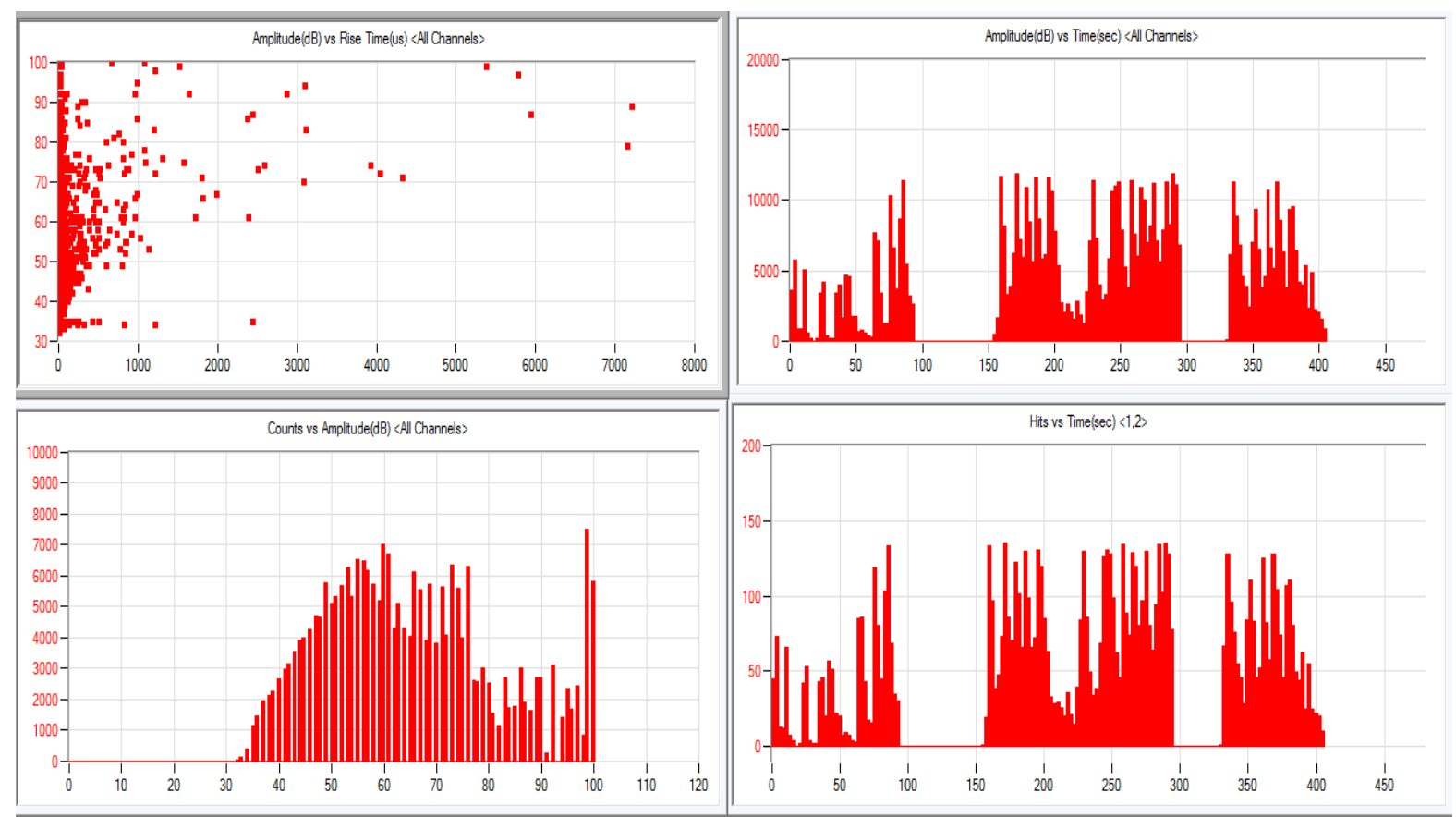

Figure 10. AE signals of the sample during dynamic Condition

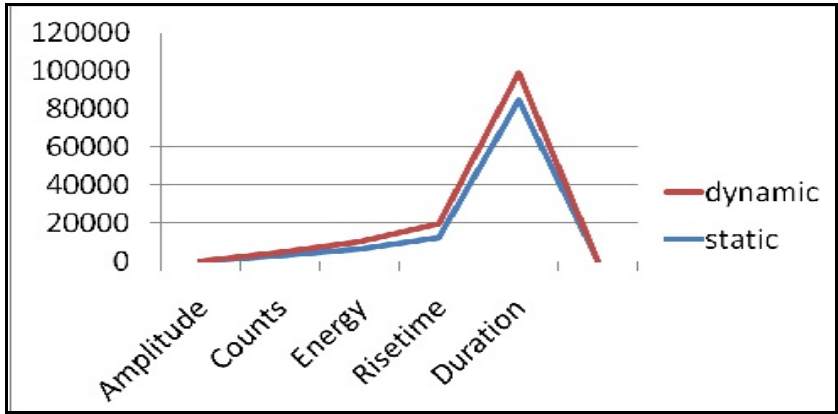

Figure 11. Analysis of the AE parameters of the sample for Static vs. Dynamic conditions
Tests are carried out by tensile samples in Dynamic Condition which resulted in emissions found from defect region. It is found that counts, energy, frequency are higher just as the amplitude (when cracked under propagation). All the experimental results show higher values than static and dynamic responses. (Fig: 11)

\section{Conclusions}

Calibration procedure and steps followed for characterization of samples using 4-channel of Acoustic 
Emission is discussed in this paper. This will help to find the structural monitoring of the metal materials during pre load conditions.

\section{Acknowledgements}

One of the Authors(S V Ranganayakulu) is grateful to Board of Research in Fusion Science and Technology of Department of Atomic Energy (Project No. NFP-MAT-F12-01) for sanction of grant-in-aid to carry out this word as part of the execution of the academic research project.

\section{REFERENCES}

[1] Breckenridge, F. Acoustic Emission Transducer calibration by means of the Seismic Surface Pulse. J. Acoustic Emission 1: 87-94; 1982.

[2] Breckenridge, F.; Greenspan, M. Surface-wave Displacement: Absolute Measurements Using a Capacitive Transducer. J. Acoust. Soc. Am. 68: 1177-1185; 1981.
[3] Hsu, N.; Simmons, j. A.; Hardy, S. An Approach to Acoustic Emission Signal Analysis- Theory and Experiment. Matls. Eval. 35: 100-106: 1977

[4] Spanner, J. C. Advances in Acoustic Emission. Dunhart: U.S.A.: 1981. P. 1.

[5] Malen, K.; Bolin, L. A Theoretical Estimate of Acoustic Emission Stress Amplitudes. Phys. Stat. sol. (b)61: $63645 ; 1974$.

[6] Stephens, R. W. B.; Pollock, A. A. Waveforms and Frequency Spectra Of Acoustic Emission. J. Acoust. Soc. Am. 50: 904-910; 1971

[7] Mura, T. Continuous Distributions of Moving Dislocations. Philos. Mag. 8: 843-857; 163.

[8] Tatro, C. A. Design Criteria For Acoustic Emission Experiment, In Acoustic Emission, ASTM STP 505, hiladelphia, PA: American Society for Testing and Materials; 1972.

[9] Hsu, N.; Eitzen, D. AE Signal Analysis- Laboratory Experiments Examining the Physical Processes Of Acoustic Emission. Proc. Fifth Int. Acoustic emission Symp., Tokyo: Japanese Society for NDI; 1980. 67-78.

[10] Jones, M.; Green, R.; Hsu, N. Comparison of Simulated Acoustic Emission Sources. Proc. Ultrasonics Int. 1983. 